\title{
Oxidative Stress and Schizophrenia
}

\author{
Dong Huang ${ }^{1}$, Shaowen Liu ${ }^{1 *}$ \\ 1 Mental Health Center of Shantou University, Shantou, Guangdong, P.R. \\ China, 515065.
}

First Author: Dong Huang, currently candidate for master's degree of Shantou University Medical College, Shantou, Guangdong, P.R. China.

* Corresponding to: Shaowen Liu. Email: liushaowen@vip.163.com.

\begin{abstract}
Many studies show that oxidative stress is a pathophysiological mechanism of schizophrenia. Elevated oxidative stress levels and the damage to the antioxidant defense system can observed in early schizophrenia. High oxidation levels damage lipids, proteins, nucleic acids and other tissues. Antioxidant defense system imbalance has been observed in both neuroleptic-naïve first-episode schizophrenics and chronic schizophrenics being treated with medication and indicates the presence of oxidative stress in schizophrenia.

Antipsychotic drugs also exert certain impacts on oxidative stress while improving the schizophrenia clinical symptoms. Antioxidants synergistically improve schizophrenia clinical symptoms. A combination regime of antipsychotic drugs and antioxidants is a potentially effective treatment strategy for schizophrenia.
\end{abstract}

Keywords: Schizophrenia; Oxidative stress; Antioxidant defense system; Antioxidants.

Schizophrenia is a common, serious mental illness, usually associated with, among other things, disordered perceptions, thoughts, emotions, volition and behavior. Major symptoms include positive symptoms (e.g. delusions or hallucinations), negative symptoms (i.e. affective flattening, alogia, or avolition) and cognitive dysfunction. The precise etiology of the disease remain unclear and both genetic and environmental factors appear to be involved. Recently scholars and researchers have begun to study the effect of oxidative stress on schizophrenia pathophysiology and it is thought to be involved in schizophrenia generation and development. This study reviews, and summarizes, schizophrenia pathogenesis, and oxidative stress medication therapy. Oxidative stress explained first, followed by a discussion of its relationship to schizophrenia. It then discusses 
oxidative stress from the perspective of the oxidative and anti-oxidative systems, and finally, it explores the synergistic effects of antipsychotic drugs and antioxidants.

\section{OXIDATIVE STRESS}

Oxidative stress results from the presence of an excess of highly reactive molecules of reactive oxygen species (ROS) and reactive nitrogen species (RNS). This presence results from harmful stimuli which generate oxides beyond the ability to remove them and leads to an imbalance between the oxidative and antioxidant systems. Tissue damage, mainly to lipids, proteins and DNA, results ${ }^{[1]}$. There are two kinds of antioxidant defense systems: 1) enzymatic anti-oxidants; and, 2) non-enzymatic antioxidants. The first is the major antioxidant defense system in cells, primarily three key enzymes: superoxide dismutase (SOD); catalase (CAT); and, glutathione peroxidase (GSH-Px). The second is the major antioxidant defense system for extracellular fluid including, among other things, plasma, and cerebrospinal fluid. It primarily involves vitamins A, C, and $E$ (tocopherol), glutathione, uric acid, albumin, and bilirubin ${ }^{[2]}$.

\section{RELATIONSHIP BETWEEN OXIDATIVE STRESS AND SCHIZOPHRENIA}

Though constituting only $2 \%$ of body weight, the brain consumes about $20 \%$ of its oxygen. It is rich in phospholipids and transitional metal ions ( $\mathrm{Fe} 2+$, $\mathrm{Cu}+$ ), but contains a relatively low level of antioxidant enzymes. Nerve tissues are extremely sensitive to ROS and RNS-caused oxidative damage. Neurons mitochondrial aerobic respiration, never cell structure component perioxidation, and microglia phagocytosis all produce large amounts of ROS and RNS causing oxidative damage to brain tissue ${ }^{[3]}$. The oxidative stress mechanism of schizophrenia is not fully understood, but increasingly more evidence supports a hypothesis that oxidative stress plays a role in schizophrenia pathophysiology.

\subsection{Elevated Indicator Level of Oxidative Stress in Schizophrenia}

Currently many studies have found the presence of elevated levels of oxidative stress in schizophrenics. Through the clinical studies of hospitalized schizophrenia selected from an average disease duration of 5 years treated with second antipsychotic drugs during therapy, DietrichMuszalska, et al. ${ }^{[4]}$ found that carbonyl groups and 3-nitrotyrosine were significantly higher in the experimental group thans the normal control group. Herken, et al. ${ }^{[5]}$ and Al-Chalabi, et al. ${ }^{[6]}$ found that erythrocyte thiobarbituratic acid reactive substances (TBARS) in chronic schizophrenics treated with stable daily doses of neuroleptics and serum malondiadehyde (MDA) in patients newly diagnosed with schizophrenia were significantly increased, respectively. Yao, et al. ${ }^{[7]}$ found, in an autopsy study, that NO levels in the caudate areas of schizophrenics in the experimental group were significantly higher than that in the normal control group. This indicates different degrees of oxidative stress in different brain areas in schizophrenics. Radonjic, et al. ${ }^{[8]}$ confirmed this finding in animal studies in which phencyclidine-fed perinatal Wister rats were used to produce schizophrenic animal models. It was found that lipid peroxidation levels in the hippocampus and thalamus areas were significantly increased. Schizophrenics are in a highly oxidated state and a variety of oxidative stress indicators are significantly elevated over that of healthy populations. Oxidation states differ from one brain area to another. Oxidation products undoubtedly damage tissue which may not the primary cause, but the damage may be a common pathogenesis for schizophrenia deterioration and poor prognoses.

\subsection{Changes of Antioxidant System in Schizophrenia}

Studies have concentrated on the enzymatic antioxidants in schizophrenia. Few have focused on non-enzymatic antioxidants. There have been studies on schizophrenia-based antioxidant enzymes but no consistent results have appeared. Some papers suggest that the level of the enzymatic antioxidants is higher, or lower, in schizophrenics than in normal controls while others have found no change. No consistent findings for the three key enzymes have been obtained either between, or within, studies. There are gender-based differences. Levels vary among schizophrenia subtypes. Studies that divide experimental patients into three subtypes: paranoid type; residual type; and disorganized type, Herken, et al. ${ }^{[9]}$ did find that erythrocyte CAT level of the three subtypes was significantly higher than that of the normal control group. However erythrocyte SOD levels were not significantly different. In addition to the paranoid type, the erythrocyte GSH-Px level of other two subtypes was higher than the normal control group. Residuals had higher erythrocyte SOD levels than paranoids. Wu, et al. ${ }^{[10]}$ discovered that 
patient plasma SOD levels of both first-episode never took drugs and administered chronic schizophrenics in the experimental group were significantly higher than the normal control group. Plasma SOD levels of the first-episode schizophrenia negatively correlated with PANSS positive subscale scores.

Most studies suggest that schizophrenia patient levels of plasma non-enzymatic antioxidants, such as uric acid, bilirubin, and albumin, were lower than that of normal controls. Some researchers found that it did not change significantly ${ }^{[10]}$. Recently, the retrospective study of total plasma bilirubin in schizophrenics by Widschwendter, et al. ${ }^{[11]}$ found that levels were significantly decreased in the second week and fourth week of treatment compared with the baseline, while the fourth week of treatment levels decreased more significantly. Meanwhile, total plasma bilirubin level changes significantly correlated with PANSS positive subscale score changes. Reddy, et al. ${ }^{[12]}$ found that levels of uric acid, bilirubin, and albumin in experimental group were significantly lower than the normal control group, and there were gender differences. The changes in these indicators in the experimental group was not significantly associated with smoking. This is consistent with most studies that have suggested that smoking did not significantly affect plasma nonenzymatic antioxidants level in schizophrenics.

There appears to be a significant heterogeneity in schizophrenic antioxidant defense systems , indicating that it may associate with the three schizophrenic subtypes, disease status, disease development stage, antipsychotic drugs use, gender, and other factors. Research results vary for different case samples. Most current studies do not strictly control related factors, and more standardized randomized controlled trials are required to clarify antioxidant defense system changes in schizophrenics. The antioxidant defense system was associated with the PANSS positive subscale score or maybe the PANSS negative subscale score, too. Some researchers have concluded that it also relates to patient cognitive symptoms ${ }^{[13]}$ and the extrapyramidal symptoms induced by antipsychotic drugs $^{[14]}$. These studies of oxidative stress indicators and antioxidant defense systems have shown that antioxidant defense system defects reflect early pathophysiologic changes in schizophrenics, rather than factors such as the results of medication, chronic disease, or smoking.

\section{EFFECTS OF ANTIPSYCHOTIC DRUGS ON ANTIOXIDANT DEFENSE SYSTEMS OF}

\section{SCHIZOPHRENIA}

Studies of haloperidol-based medications are in a majority among typical antipsychotic medications with studies of atypical antipsychotic medications such as, risperidone, quetiapine, olanzapine, ziprasidone forming the balance. Al-Chalabi, et al. [6] showed that olanzapine could improve plasma total antioxidant status (TAS) and alleviate lipid peroxidation damage. Eftekhari, et al. ${ }^{[15]}$ found, in animal experiments, that olanzapine induced oxidative stress and hepatocytotoxicity and associated with the CYP450 enzyme. Olanzapine and clozapine were recently thought to be more effective than risperidone, quetiapine, ziprasidone and haloperidol in resisting oxidation ${ }^{[16]}$. For the effects of typical and atypical antipsychotic drugs on oxidative stress, typical neuroleptics may aggravate oxidative damage as the resulting extrapyramidal side effects are thought to be associated with typical antipsychotic drugs-based oxidative damage. On the contrast, atypical antipsychotic drugs may lessen oxidative damage. Whether this is a direct, or indirect, effect warrants further study. The different research results of antipsychotic drugs suggests that schizophrenic oxidative stress may be independent of antipsychotic medication therapies and reflect the pathophysiological progress of the disease.

\section{EFFECTS OF ANTIOXIDANTS ON SCHIZOPHRENIA}

Some researchers have further analyzed the effects of oxidative stress on the schizophrenia by focusing on antioxidants. Studies have concentrated on medications such as $\mathrm{N}$-acetylcysteine (NAC), ginkgo biloba extracts (EGb), $\omega-3$ fatty acids, and vitamins $C$ and $E$. Farokhnia, et al. ${ }^{[17]}$ found that a combination of antipsychotic medications with NAC significantly improved schizophrenics negative symptoms while no significant effects were found in improving either positive symptoms or general psychopathology symptoms (based on the changes of the PANSS total scores and its three subscale scores). Jingfeng Qi, et al. ${ }^{[18]}$ found, in a psychiatric study, that $\omega-3$ fatty acids increased antioxidant enzyme levels and alleviated oxidative stress in the brain, was synergistic with antipsychotic medications, improved PANSS total scores, and separately improved schizophrenic symptoms. Synergistically, antioxidants played a certain therapeutic role in schizophrenia treatment, suggesting that they may be considered a possible new schizophrenia treatment strategy. However, the pharmacological mechanisms and applicable conditions and other limitations should be further studied. 


\section{CONCLUSION}

The etiology of schizophrenia is remains unclear. It has begun to be considered a progressive neurodevelopmental disorder as there are progressive abnormal changes in brain structure and brain function. A variety of possible pathological mechanisms, such as neurobiological pathology hypothesis, have been proposed. More and more evidence suggest that there is oxidative stress effect in the pathophysiology of schizophrenia, that there is an imbalance between oxidant system and antioxidant defense system that results in tissue damage, an increase in oxidation and damage to the antioxidant defense system. Multiple oxidative stress indicators levels were significantly higher in schizophrenics than in normal controls, and the level of both its enzymatic antioxidants and non-enzymatic antioxidants was significantly different to normal controls, some of enzymatic antioxidants levels are higher or lower, most of non-enzymatic antioxidants levels are lower than that of normal controls. Antioxidant defense system changes associated with clinical symptoms of schizophrenia and with disease development and prognosis. They also associated with disease severity, disease status, social demographics and other factors. Current studies do not provide definitive evidence of whether the disease state or antipsychotic medications are more closely related to oxidative stress. Future studies should strictly control the relevant factors to assure more meaningful and valuable results.

\section{REFERENCES}

1. Valko M, Leibfritz D, Moncol J, Cronin MT, Mazur $M$, Telser J. Free radicals and antioxidants in normal physiological functions and human disease. Int J Biochem Cell Biol. 2007; 39(1): 44-84.

2. Halliwell B, Gutteridge JM. The antioxidants of human extracellular fluids. Arch Biochem Biophys. 1990; 280(1): 1-8.

3. Moniczewski A, Gawlik M, Smaga I, Niedzielska E, Krzek J, Przegaliński E, Pera J, Filip M. Oxidative stress as an etiological factor and a potential treatment target of psychiatric disorders. Part 1. Chemical aspects and biological sources of oxidative stress in the brain. Pharmacol Rep. 2015; 67(3): 560-568.

4. Dietrich-Muszalska A, Malinowska J, Olas B, Głowacki R, Bald E, Wachowicz B, RabeJabłońska J. The oxidative stress may be induced by the elevated homocysteine in schizophrenic patients. Neurochem Res. 2012; 37(5): 1057-1062.

5. Herken H, Uz E, Ozyurt H, Söğüt S, Virit O, Akyol $O$. Evidence that the activities of erythrocyte free radical scavenging enzymes and the products of lipid peroxidation are increased in different forms of schizophrenia. Mol Psych. 2001; 6(1): 66-73.

6. Al-Chalabi BM, Thanoon IA, Ahmed FA. Potential effect of olanzapine on total antioxidant status and lipid peroxidation in schizophrenic patients. Neuropsychobiol. 2009; 59(1): 8-11.

7. Yao JK, Leonard S, Reddy RD. Increased nitric oxide radicals in postmortem brain from patients with schizophrenia. Schizophr Bull. 2004; 30(4): 923-934.

8. Radonjic NV, Knezević ID, Vilimanovich U, Kravić-Stevović T, Marina LV, Nikolić T, Todorović V, Bumbasirević V, Petronijević ND. Decreased glutathione levels and altered antioxidant defense in an animal model of schizophrenia: long-term effects of perinatal phencyclidine administration. Neuropharmacol. 2010; 58(4-5): 739-745.

9. Wu Z, Zhang $X Y$, Wang $H$, Tang $W$, Xia $Y$, Zhang F, Liu J, Fu Y, Hu J, Chen Y, Liu L, Chen DC, Xiu MH, Kosten TR, He J. Elevated plasma superoxide dismutase in first-episode and drug naive patients with schizophrenia: inverse association with positive symptoms. Prog Neuropsychopharmacol Biol Psych. 2012; 36(1): 34-38.

10. Sarandol A, Sarandol E2, Acikgoz HE2, Eker SS1, Akkaya C1, Dirican M2. First-episode psychosis is associated with oxidative stress: Effects of short-term antipsychotic treatment. Psychiatry Clin Neurosci. 2015; 69(11): 699-707.

11. Widschwendter CG, Rettenbacher MA, Kemmler G, Edlinger M, Baumgartner S, Fleischhacker WW, Hofer A. Bilirubin concentration correlates with positive symptoms in patients with schizophrenia. J Clin Psych. 2016; 77(4): 512516.

12. Reddy R, Keshavan M, Yao JK. Reduced plasma antioxidants in first-episode patients with schizophrenia. Schizophr Res. 2003; 62(3): 205212.

13. Zhang XY, Chen DC, Xiu MH, Tang W, Zhang F, Liu L, Chen Y, Liu J, Yao JK, Kosten TA, Kosten TR. Plasma total antioxidant status and cognitive impairments in schizophrenia. Schizophr Res. 2012; 139(1-3): 66-72. 
14. Boskovic M, Grabnar I, Terzič T, Kores Plesničar B, Vovk T. Oxidative stress in schizophrenia patients treated with long-acting haloperidol decanoate. Psychiatry Res. 2013; 210(3): 761768.

15. Eftekhari A, Azarmi Y, Parvizpur A, Eghbal MA. Involvement of oxidative stress and mitochondrial/lysosomal cross-talk in olanzapine cytotoxicity in freshly isolated rat hepatocytes. Xenobiotica. 2016; 46(4): 369-378.

16. Brinholi FF, Farias CC, Bonifácio KL, Higachi L, Casagrande R, Moreira EG, Barbosa DS. Clozapine and olanzapine are better antioxidants than haloperidol, quetiapine, risperidone and ziprasidone in in vitro models. Biomed Pharmacother. 2016; 81: 411-415.

17. Farokhnia M, Azarkolah A, Adinehfar F, KhodaieArdakani MR, Hosseini SM, Yekehtaz H, Tabrizi M, Rezaei F, Salehi B, Sadeghi SM, Moghadam M, Gharibi F, Mirshafiee O, Akhondzadeh S. $\mathrm{N}$-acetylcysteine as an adjunct to risperidone for treatment of negative symptoms in patients with chronic schizophrenia: a randomized, double-blind, placebo-controlled study. Clin Neuropharmacol. 2013; 36(6): 185-192.

18. Qi JF, Guo XF, Zhao JP. Application Progress of w-3 Fatty Acids in Psychiatry. J Int Psych. 2015; 1: 126-129. 